\title{
Placement of replace select Ti-Unite-coated type implants using a combination of immediate and submerge techniques after tooth extraction
}

Coen Pramono D

Department of Oral and Maxillofacial Surgery

Faculty of Dentistry Airlangga University

Surabaya - Indonesia

\begin{abstract}
The high success rate of immediate implant placement both in the anterior and posterior regions were reported by many authors, therefore applying this techniques can be considered as a safe surgical procedure and minimizing the dental office visit for patient satisfaction. This paper reports the outcome of immediate placement of implants following extraction of anterior maxillary teeth. Combination technique of immediate and submerge implant placements including bone grafting procedure were used. Four implants with TiUnite surface type were placed immediately in two patients with the short-term result indicated that this technique may serve as a simple and safe procedure for immediate implant placement. It was concluded that immediate implant placement technique combined with TiUnite implant surface was successful in treating region directly after tooth extraction therefore this technique can be use as an alternative surgical method for dental implant rehabilitation.
\end{abstract}

Key words: immediate implant placement, Ti-Unite-coated implant surface, osseointegration

Correspondence: Coen Pramono D, c/o: Bagian Bedah Mulut, Fakultas Kedokteran Gigi Universitas Airlangga. Jln. Mayjend. Prof. Dr. Moestopo No. 47 Surabaya 60132, Indonesia.

\section{INTODUCTION}

In May 1982 conference held in Toronto, the North American Dental profession was introduced to a body of scientific literature on Swedish research into bone-to implant interface, a concept called osseointegration. This concept is based on a-traumatic implant placement and delayed implant loading. The Swedish research team led by PI Branemark reported the high success of implant placement in the mandible over 15 years. ${ }^{1}$

The recent success of dental implants relates directly to the discovery of method to maximize the amount of bone and implant contact.

Extraction and immediate use of immediate denture is common solution to aesthetic problem. However, the subsequent healing of the extraction socket may result in collapse of the local bone and soft tissue, leading in turn to poor esthetic outcome. Acceptable esthetic outcomes after augmentation is uncertain, another treatment option as suggested by Schwartz-Arad and Chaushu in $1997^{2}$ and Kirketerp et al. ${ }^{3}$ placement of implant combined with tooth extraction has been demonstrated as a viable option.

Pedlar and Frame 4 disagreed with immediate implant placement as the prognosis for successful osseointegration is poor, especially in the presence of apical pathology prior implant insertion. Other problems likely to be encountered including the differences between the width of the natural tooth and the endosseous implant and insufficient bone at the coronal aspect, therefore a bone graft may be required to close the defect. A further disadvantage is that nonkeratinized mucosa may have to be advanced to cover the implant. They suggested that 5 to 6 weeks after tooth extraction, the soft tissue will have healed but the residual ridge should not have undergone excessive resorption.

The recent success of dental implants relates directly to the discovery of method to maximize the amount of bone and implant contact. Osseointegration is a histologic definition meaning as: a direct contact between living bone and loaded-bearing endoseous implant at the microscopic level. ${ }^{1}$ This concept of osseointegration has brought a revolutionary progress of dental implantology in dentistry.

Placement of 36 implants Replace Select HA immediately after teeth extraction reported by Kirketerp et al. ${ }^{3}$ showed that 35 of 36 immediately replaced maxillary front teeth were clinically stable and asymptomatic after one year loading. Whorle in 1998 reported immediate replacement of 14 extracted teeth in the anterior maxilla. All implants remained stable during the 9 to 36 months follow-up. ${ }^{4}$

Glauser et al. ${ }^{5}$ and Rocci et al. ${ }^{6}$ reported the excellent clinical outcome of immediately loaded partial fixed bridge. Glauser et al. ${ }^{5}$ reported success rate of $97 \%$ following immediate load of TiUnite implant placed predominantly in soft bone and implant placed in bone quality 4 showed a $100 \%$ success rate. 6 Study by Rocci et al. ${ }^{6}$ comparing between immediate loading of TiUnite and machined implants in the posterior mandible, a $10 \%$ higher success rate was found for TiUnite implants $(95.5 \%)$. The present 
histological evaluation confirmed that successful osseointegration of TiUnite implant placed in soft bone is possible when the implant is immediately loaded. The $93.3 \%$ bone-implant contact achieved might even imply the immediate loading had stimulated the bone healing process.

Recently an implant surface modification has been developed for enhancing early bone response and this surface has been shown to enhance primary implant stability, 7,8 and to achieve secondary stability earlier than a machined surface. ${ }^{9,10}$

\section{CASES}

Two cases of patients treated with immediate implant placement in the anterior regions of the upper jaw are reported. Those two patients are 41 year old man presented in case 1 and 25 year old women presented in case 2 . Both patients asked for dental implant treatment as they refused for a fixed crown and bridge denture rehabilitation after their teeth being extracted. Both patients also have limited time for having their dental treatment, therefore they asked for the possibility of an alternative treatment used a direct implant placement after teeth extractions for time saving.

The treatment planning included measurement of implant depth using Coen's measurement technique, ${ }^{11}$ a-traumatic tooth extraction using periotome, careful cleaning of the socket and placement of the implant with raising a flap. Due to the bone available after teeth extraction an implant loading were delayed for a minimum period of 2.5 months after implant placement. Torch of $35 \mathrm{Ncm}$ were un-reached in both cases as the alveolar bone condition had mostly resorbed by an inflammatory processes, but relative primary implant stability was achieved as the implants were inserted more palatal and an additional bone stability was taken by passing the implants slightly through the alveolar apical bone area to reach a healthy and compact bone.

Replace select TiUnite from Nobel biocare dental implants were used in these cases, one dental implant was inserted in case 1 and three in case 2 . In both cases removable partial dentures were prepared pre-operatively for cosmetic reason, designed with freed in the cervical area to avoid soft tissues pressure in the implant sites during its use after the implants had been placed.

\section{CASE MANAGEMENT}

Case 1: A 41 year old male complained of recurrent abscesses in the labial site of tooth 12 (Figure 1-a). Panoramic radiograph presented with a radiolucent area surrounded the apical region of tooth 12 almost closed to the alveolar crest (Figure 1-b). Preservation of that tooth with root resection was presumed with un-successful result, therefore tooth extraction followed by immediate implant placement was chosen as a choice of treatment as the patient refused for a fixed crown and bridge denture. Intraoperative steps were done with extraction of tooth 12 (Figure 1-c) and a wide base mucoperiosteal flap was made and deflected (Figure 1-d). The cyst and the remnants soft tissue were curettage and a thin labial bone was carefully preserved to be intact (Figure 1-e). Step by step bone drilling was made with initial direction more to the palatal bone and afterward the drilling angle direction changed parallel to the axis of adjacent anterior teeth (Figure 1-f). The drilling process continued into the required implant depth and diameter. A $4.3 \mathrm{~mm}$ TiUnite implant from Nobel Biocare was placed, some gaps around the implant sites were showed therefore a bone graft which had taken from the region of right zygoma was grafted, the mucosa then sutured back covered the whole implant body. The implant abutment was connected to the implant body 5 months after implant placement and followed by single ceramic crown rehabilitation (Figure 1-h and i). No clinical symptom was reported by the patient. Clinical observation 5 months after implant placement showed the implant had been reached its stability in satisfactory (Figure 1b-i).

Case 2: A 25 year old female with chief complaint of pain sensation in the apical region of teeth 11,21 and 22 asked for dental treatment. The patient had undergone for apical teeth resection on those teeth 5 years before. Radiographic examination showed with apical radiolucent on those three teeth, and had an indication of teeth extraction (Figure 2-a). Immediate implant placement was planned for teeth replacement. TiUnite implant from Nobel Biocare with $5.0 \mathrm{~mm}$ diameter for teeth 11 and 21 and 4.3 $\mathrm{mm}$ diameter for tooth 22 were used. Pre-operatively a removable denture was prepared for cosmetic consideration. The teeth 11, 21 and 22 were extracted carefully and a wide based mucoperiosteal flap was made with posterior incision sides at the distal ends of the extracted teeth. A deep curettage was done to clean the socket from cyst and remnants tissues. The labial bone was carefully maintained to avoid from bone lost. Step by step drilling for $4.3 \mathrm{~mm}$ and $5.0 \mathrm{~mm}$ diameter dental implants were made and left the labial bone intact, and followed by implant body placement. Up to one week showed all implants were free from clinical symptoms and inflammatory process. Panoramic radiograph ten days after implant placement showed all implants were well placed (Figure 2-b).

Clinical and panoramic radiography observation 2.5 months after three implant placement showed with good results (Figure 2-c). Clinical symptoms as pain, implants mobility and sign of infection were not presented. A-traumatic gingival opening using punch technique was done for exposing those three dental implants (Figure 2-d). Fifteen degree type of abutment was connected to implant bodies in teeth 11 and 21, and a normal abutment in tooth 22 and screwed until the $15 \mathrm{Ncm}$ torch had reached and three single ceramic crowns were inserted by her private dentist (Figure 2-e-g). 

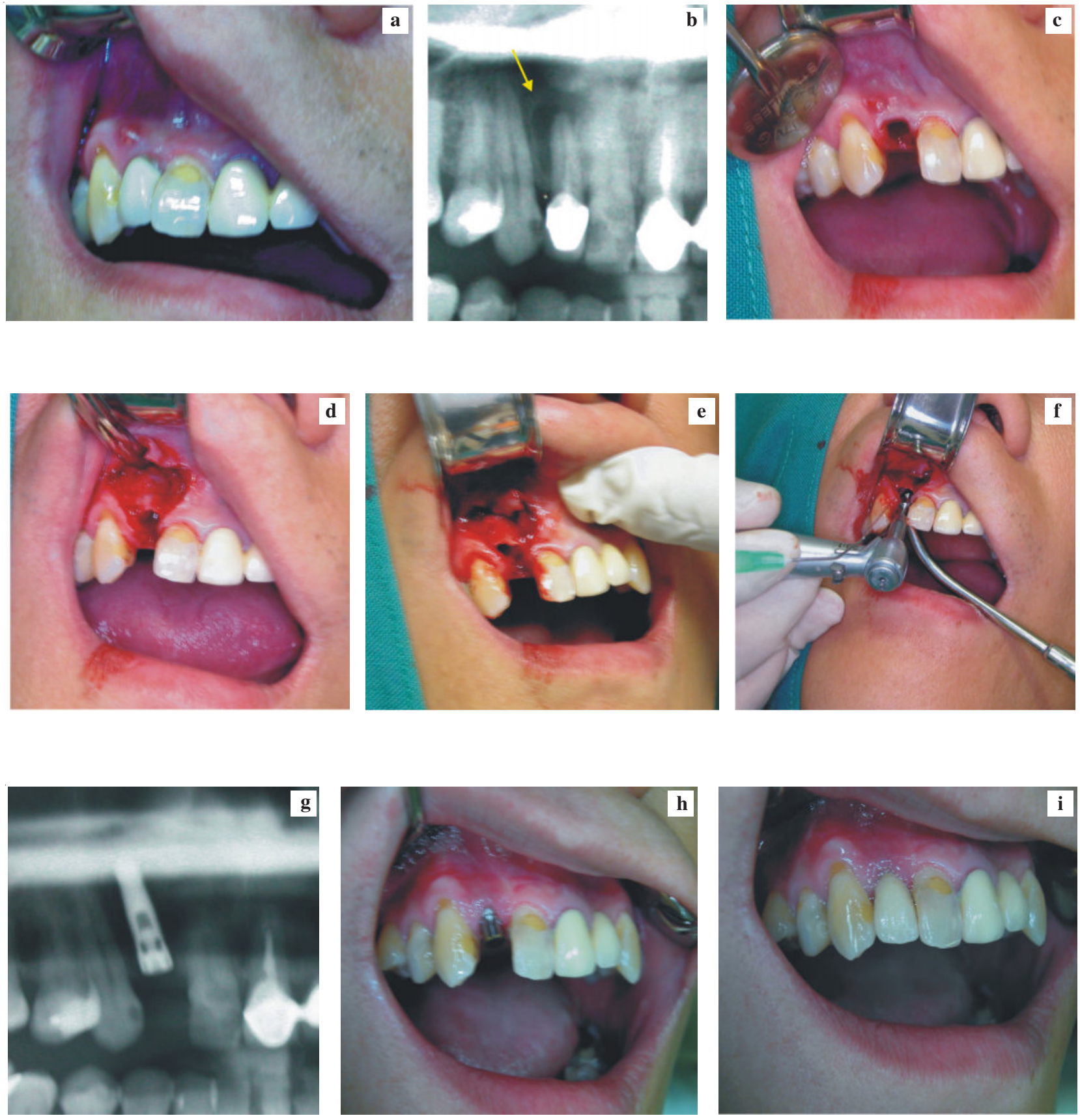

Figure 1. (a) Tooth 12 with recurrent of gingival abscess.

(b) Periapical cystic lesion grown almost involving the entire distal alveolar crest of tooth 12 .

(c) Tooth extracted left good dental socket.

(d) Flap deflected and the socket deeply curettage.

(e) Socket curettage and left the thin labial alveolar bone intact. (f) Step by step direct bone drilling on the fresh socket for implant immediate placement and left the labial-bone un-disturb.

(g) Panoramic x-ray:5 months after implant placement. A new bone grown seen fulfill the bone defect.

(h) The abutment fixed on the implant.

(i) Final single ceramic crown situation 6 months after implant placement. 

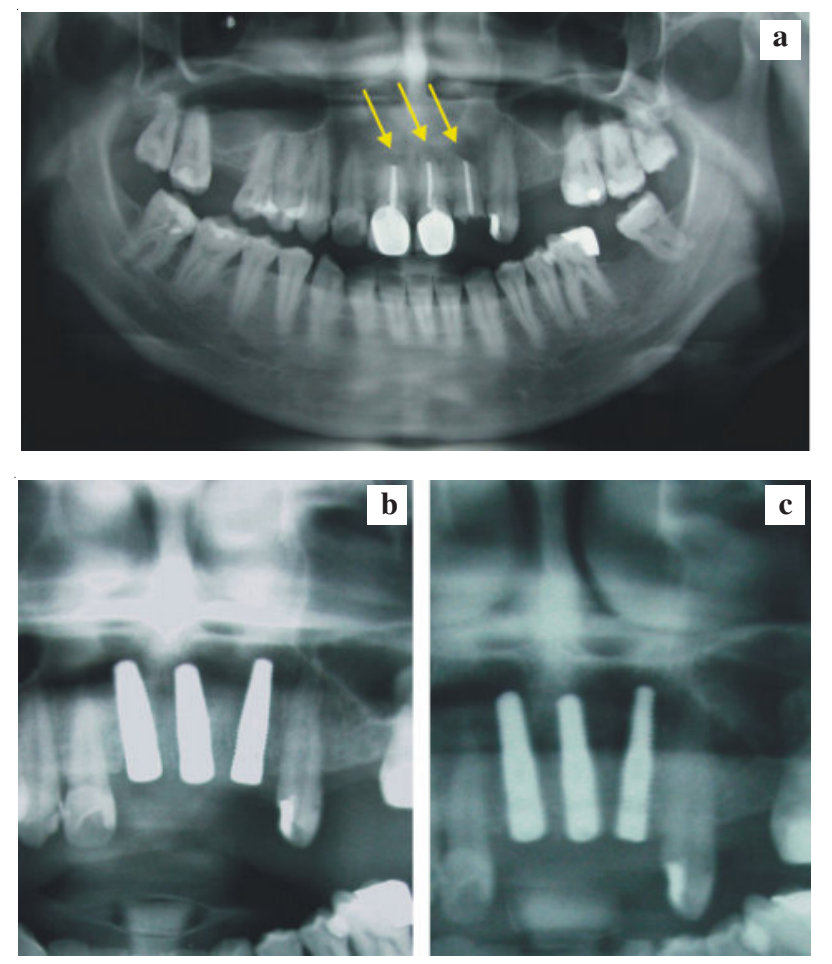
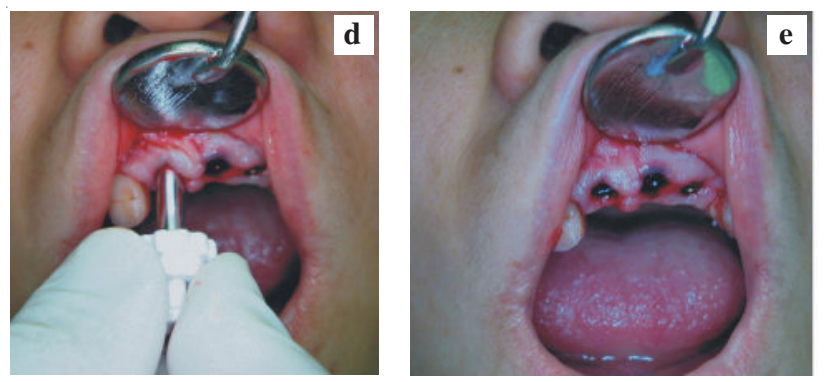

Figure 2. (a) Pre-operative panoramic radiography view: teeth 11,21 and 22 with apical radiolucent post roots resection.

(b) Enlarged panoramic radiography 10 days after implant placement.

(c) Two and a half months after days implant placement.
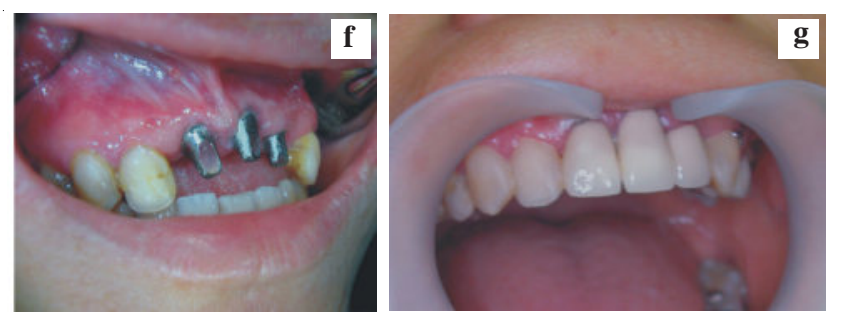

(d) A soft tissue punch method gives a-traumatic gingival opening.

(e) Three stable implants 2,5 months after insertion and exposed a-traumatically.

(f) Situation 4 days after implants exposure and connection of fifteen degrees abutment in teeth 11,21 and straight form abutment in tooth 22 .

(g) Final single ceramic crowns on teeth 11,12 and 22 .

\section{DISCUSSION}

Immediate implant placement was performed based on considerations that placement of implant and waiting for the bone has to be matured which usually taken about 3 to 6 months would be time consuming. The high affinity and degree of bone formation promoted into the implant surface due to the available modified implant surface with anodic oxidation technique had proved by some authors with significant osseointegration result, ${ }^{3,5-12}$ therefore the success of those cases reported had been taken as a basic knowledge on predicting the success these implant placement.

Implant placement need to be inserted in a good bone quality, and dental implant usually being performed after the bone had consolidated which can be found in the range of time about 3 to 12 months after tooth extraction. Some authors had reported a good result of direct implant placement into a good bone quality and directly loaded with direct loading. In such situation implant placement needed to be placed immediately as patient has a limited visiting time to visit the dental office. Direct implant placement immediately after tooth extraction can be used as an alternative method then placed waiting for the bone had reached its maturity. 
Two cases of immediate dental implant placement are reported. Implant placement in the extraction socket often leads no primary bone contact surrounding the implant body. If the implant stability is achieved, it is usually due to bone contact at the palatal bone side and in the apical end of the socket. The coronal end and some part of the labial site usually can be found with slightly open or even the implant threads might be found exposed due to the implant diameter is smaller than the tooth size which needed to be replaced. Therefore bone graft is usually necessary to be placed around the implant body necessary to cover some of the exposed implant with bone graft.

Bone integration of such an implant depends on bone being formed from adjacent bone surfaces toward the implant surface. Histological evidences were found by Carlsson et al. and cited by Salata et al. ${ }^{12}$ and Akimoto et al. ${ }^{13}$ that large gaps between a turned implant and bone will not be filled with bone. Clinical study by SchwartzArad and Chaushu ${ }^{2}$ presented a good long-term result of placing implants into fresh socket. In this surgical report, in both cases bone graft procedure were done, as some gaps were presented between bone-implant.

In these cases the bone-implant gaps were harvested with an autogenous bone which had taken from the bone residues during bone drilling process and added with some bones taken from the zygoma area. This bone graft procedures were performed to enhance the process of osseointegration. A mucosa tissue split technique for bone covering was done to stabilize the harvested bone graft material remain stable in the grafted areas. The periosteum was separated from the mucosa, adapted and sutured around the neck implants, followed by mucosa adaptation and suturing.

A recent study found that the junctional epithelium effectively attached via hemidesmosome to uniquely porous TiUnite surface much like natural teeth. The connective tissue interposed between junctional epithelium and the alveolar crest is dominated by both circumferentially and longitudinally running collagen fibers. As a result, the connective tissue around TiUnite implants is stable and healthy. ${ }^{14}$

A number of studies in implant type which its surface modified by anodic oxidation have shown a higher affinity and degree of bone formation. ${ }^{9,10}$ A study by Salata et al. ${ }^{12}$ using Resonance Frequency Analysis on (RFA) NP MkIII TiUnite, Nobel Biocare AB supported the previous studies. ${ }^{9,10}$ They concluded that oxidized implant surface has a significant influence on bone formation when placed in bone defects and the implant stability increase more rapidly.

The excellent clinical outcome of this direct implant placement reported in this case is nearly closed with the data reported by Glauser et al. ${ }^{5}$ A different situation given in these case reported, an immediately implant loaded was considered to be too risky to performed as only a thin part of the labial bone left intact and the maximum implant primary stability of 30 to $35 \mathrm{~N} / \mathrm{cm}^{2}$ was not achieved, therefore implant placement was done in combination, direct implant placement immediately after tooth extraction and submerged technique. Two and a half months after the implant placement, good stability is shown in all 4 implants observed. This situation can be assumed that the 4 walls bone of the dental sockets had grown and the implantsbones osseointegration had achieved properly.

Some critical steps during the surgical procedure were noted as: 1) the bone gaps between implants and bones, therefore bone grafting was considered necessary to be applied to close those gaps, 2) during the drilling procedures highly consideration should be mentioned: a) the initial drilling direction must be directed to the palatal wall direction of the post extraction socket to achieve more compact bone for implant placement in achieving primary stability and b) change of the drill's trajectory from palatal into parallel direction with the adjacent teeth to achieve good implant axis. This step can be paid a consequence while it related with the result of implant angulations. As shown in case 2, two implants were placed in the region of teeth 21 and 22 with the implants axis position more palatal, a 15 degree angled abutments was necessary to be used to compensate the implant position.

According to my early experience can be concluded that combination of immediate and submerge implant placement technique and additionally with bone grafting procedure to cover the uncovered implant surface presented constitutes a simple and safe procedure for replacement of hopeless anterior teeth in the maxilla. Application of this technique gave a favorable experience as the implant can be placed with clear surgical axis angulations following the alveolar socket of the extracted teeth. Additionally bone stability can be achieved by placement the dental implant slightly to the palatal bone and passing through the implants end to the alveolar apical bone area to reach a healthy and compact bone.

\section{REFERENCES}

1. Peterson LJ, Ellis E, Hupp JR, Tucker MR. Contemporary oral and maxillofacial surgery. $4^{\text {th }}$ ed. St Louis: Mosby; 2003. p. 305-42.

2. Schwartz-Arad D, Chaushu G. The way and where forces of immediate placement of implants into fresh extraction sites: A literature review. J Periodontol 1977; 68(9):15-23.

3. Kirketerp P, Andersen JB, Goeran U. Replacement of extracted anterior teeth by immediately loaded replace select HA-coated implants. A one-year follow-up of 35 patients. Appl Osseointegration Res 2002; 3(1):40-3.

4. Pedlar J, Frame JW. Oral and maxillofacial surgery. An objectivebased text book, Churchill Livingstone. Printed in Spain. 2001. p. 157.

5. Glaucer R, Gottlow J, Lundgren AK, Senneerby L, Portmann M, Rusthaller P, Haemmerle CHF. Immediate occlusal loading of Branemark MkIV TiUnite implants placed in bone quality 4. Appl Osseointegration Res 2002; 3:22-4.

6. Rocci A, Martiggoni M, Sannerby L, Gottlow J. Immediate loading of a Branemark system implant with the TiUnite surface. Histological evaluation after 9 months. Appl Osseointegration Res 2002; 3(1): 25-8. 
7. Rompen E, DaSilva D, Lundgren AK, Gottlow J, Sennerby L. Stability measurements of double-threaded titanium implant design with turned or oxidized surfaces. An experimental resonance frequency analysis study in dog mandible. Appl Osseointegration Res 2000; 1:18-20.

8. Glauser R, Portmann M, Ruhstaller P, Lundgren AK, Haemmerle C, Gottlow J. Stability measurements of immediately loaded machined and oxidized implants in the posterior maxilla. A comparative clinical study using resonance frequency analysis. Appl Osseointegration Res 2001; 2:27-9.

9. Albrektsson T, Johansson C, Lundgren AK, Sul Y, Gottlow J. Experimental studies on oxidized implants. A histomorphometrical and biomechanical analysis. Appl Osseointegration Res 2000; 1: $21-4$.

10. Henry P, Tan A, Allan B, Hall J, Johansson C. Removal torque comparison of TiUnite and turned implants in greyhound dog mandible. Appl Osseointegration Res 2000; 1:15-7.
11. Pramono C. Introducing a simple innovative technique in achieving implant parallelism and an equation concept in measurement the discrepancy in panoramic radiograph. Accepted for publication in the J Oral Maxillofac Surg for 2006.

12. Salata L, Rasmusson L, Novaes Jr A, Papalexiou V, Sennerby L. The influence of anodic oxidation on implant integration and stability in bone defects. An RFA study in dog mandible. Appl Osseointegration Res 2002; 3(1):32-4.

13. Akimoto K, Becker W, Persson R, Baker DA, Rohrer MD, O'Neal RB. Evaluation of titanium implants placed into stimulated extraction sockets: A study in dogs. Int J Oral Maxillofac Implants 1999; 14: $351-60$.

14. Glauser R, Schuepbach P, Gottlow J, Lundgren AK, Ruhstaller P, Haemmerle CHF. Soft tissue barrier at non-submerged one-piece micro-implants with different surface topography retrieved from human, Poster presentation: $12^{\text {th }}$ annual meeting European Academy of Osseointegration, October 9-11, Viena 2003. Nobel Biocare, Nobel Direct Biologic One-piece Implant. Clinical Procedure and Product Catalog, 2004. 